\title{
ANDREAS LÖTSCHER \\ Gesetze als Texte: Wie wird Recht in Textstrukturen gebracht?
}

\begin{abstract}
1. Einleitung
Der Staat vermittelt Recht in Gesetzestexten. Sprachlich offenbar nicht immer zur Zufriedenheit der Bürgerinnen und Bürger. Gesetze gelten notorisch als schwer verständlich. Die Ursachen dafür sind vielschichtig, die Gründe bei aller Plausibilität der Einzelkritik nicht immer ganz einfach zu durchblicken. In der linguistischen Diskussion werden Probleme vor allem auf der Ebene des Wortschatzes und des Satzbaus gesehen: in der Kompliziertheit und Länge der Sätze, im ungewohnten Gebrauch der Wörter, im intransparenten Gebrauch von Pronomen, in der Unpersönlichkeit der häufigen Passivkonstruktionen. ${ }^{1}$ Die Textstruktur bleibt gewöhnlich außer Acht. Gesetze weichen aber in der äuBeren Darbietung und im Aufbau in vielem markant von gewohnten Sachtexten $\mathrm{ab}$. Ob dies einen Einfluss auf die Rezeption hat, wird gewöhnlich nicht gefragt. Im Gegenteil, in der Rechtslinguistik werden, scheint es, für die Textstrukturierung, das Verstehen und die Verständlichkeit von Gesetzestexten genau die gleichen generellen Faktoren und Bedingungen vorausgesetzt wie für beliebige andere Texte. ${ }^{2}$
\end{abstract}

\footnotetext{
1 Vgl. etwa INGEBORG LASSER, Verständliche Gesetze - eine Utopie?, in: Zeitschrift fïr Literaturwissenschaft und Linguistik 118 (2000), 34-66. In der Mehrzahl der Fälle, in denen über die Unverständlichkeit von Gesetzestexten räsoniert wird, fehlt allerdings eine systematischere Analyse der konkreten Eigenschaften, die zur Schwerverständlichkeit führen; entweder werden Einzelbeispiele angeführt oder die Unverständlichkeit wird als unhinterfragbare Eigenschaft vorausgesetzt. Als typisches Beispiel sei die Sammlung von Stellungnahmen ("Arena”) im Rechtshistorischen Journal 20 (2001), 479 ff. erwähnt (in der übrigens aber auch ein unhinterfragter Begriff der Verständlichkeit an sich problematisiert wird). Das Phänomen der Unverständlichkeit wird in dieser Diskussion allerdings auch oft als das Problem thematisiert, dass in der juristischen Auslegung Gesetzesbestimmungen einen Sinn bekommen, den der unbefangene Leser/die Leserin in keiner Weise aus dem Wortlaut ableiten würde. Das ist aber das Problem der Auslegung, das nicht zum Thema dieses Aufsatzes gehört.

2 Beispielsweise setzen Lasser oder Dietrich \& Kühn ohne weitere Einschränkungen voraus, dass die gängigen psycholinguistischen Modelle des Textverstehens, die normalerweise an einfachen deskriptiven Texten erarbeitet worden sind, tel quel auch für Ge-
} 
Ist dies angebracht? Ohne eine gründlichere Analyse der Textualität von Gesetzestexten kann diese Frage nicht ohne weiteres bejaht werden. Die nachfolgenden Überlegungen wollen dazu beitragen, in diesem Punkt mehr Klarheit zu erhalten. Gefragt werden soll: Welches sind die Vertextungsprinzipien für Gesetze, und warum sind Gesetze als Texte so strukturiert, wie sie sind? Wodurch unterscheiden sich Gesetze als Texte strukturell von anderen Texttypen? Was ergibt sich daraus für die Rezeption und das Verstehen von Gesetzestexten?

Diese Fragen werde ich vornehmlich anhand des mir vertrauten Schweizer Bundesrechts behandeln. Die Probleme stellen sich aber meiner Ansicht nach für andere Rechtssysteme westeuropäischer Rechtstradition in gleichartiger Weise. ${ }^{3}$

\section{Rahmenbedingungen für die Textsorte "Erlass"}

\subsection{Was ist eine Regelung?}

Textsorten werden unter anderem durch ihre dominierende Funktion charakterisiert. Die generelle Funktion eines Erlasses - eines Gesetzes oder einer Verordnung ${ }^{4}$ - besteht darin, für das staatliche, gesellschaftliche, wirtschaftliche usw. Handeln in einem bestimmten Sachbereich unter einem bestimmten Aspekt Regelungen zu treffen. ${ }^{5}$ Es geht dabei nicht nur um Gebote oder Verbote, also Festlegungen, was jemand tun muss oder nicht tun darf, sondern auch um die Einrichtung von Institutionen, die ein Staat braucht, um seine Aufgaben zu erfüllen, um die Regelung von Handlungsmöglichkeiten für staatliche Institutionen, um Ansprüche von Bürgerinnen und Bürgern an eine staatliche Institution oder an eine Drittperson und anderes mehr. Die Vielfalt der Regelungs-

setzestexte übernommen werden können; vgl. LASSER, Verständliche Gesetze - eine Utopie? (Fn. 1), 51, und RAINER DIETRICH / KATJA KÜHN, Transparent oder verständlich oder wie was verstanden wird, in: Zeitschrift fiir Literaturwissenschaft und Linguistik 118 (2000), 67-95, $68 \mathrm{ff}$.

${ }^{3}$ Nicht zur Diskussion steht in diesem Rahmen die Frage der Auslegung von Gesetzesbestimmungen, die eine ganz andere Problematik berührt.

${ }^{4}$ Der Ausdruck „Erlass" wird im Folgenden als Oberbegriff für alle von einer staatlichen Behörde erlassenen generell-abstrakten normativen Texte verwendet. Im Einzelnen fallen darunter Gesetze, d. h. vom Parlament erlassene Erlasse, und Verordnungen, d. h. von einer Exekutivbehörde erlassene Erlasse. Gesetze und Verordnungen folgen grundsätzlich den gleichen Textsortenregeln.

5 Im Folgenden werden unter "Regelungen" die Inhalte, unter "Bestimmungen" deren sprachliche Realisierung verstanden. 
typen ist größer als von der Rechtslinguistik im Allgemeinen wahrgenommen wird.

Sprachtheoretisch ausgedrückt ist eine Gesetzesbestimmung eine normative Aussage einer staatlichen Institution; im Fall von Erlassen handelt es sich juristisch ausgedrückt um sog. "generell-abstrakte" Normen: Bestimmungen, die sich an einen unbestimmten Adressatenkreis richten und eine unbestimmte, intensional definierte Menge von Sachverhalten regeln. ${ }^{6}$ Eine abstrakte logisch-semantische Charakterisierung der pragmatischen und illokutiven Aspekte greift allerdings als Inhaltsbestimmung in mehrfacher Hinsicht zu kurz. Warum eine Institution befugt ist, solche Regelungen bestimmten Personen und Institutionen aufzuerlegen, welches die Konsequenzen einer Regelung für Rechtsbetroffene sind, wie dafür gesorgt wird, dass Regelungen eingehalten werden, dies ist letztlich nicht in linguistischen oder sprachtheoretischen Kategorien $\mathrm{zu}$ beschreiben, sondern aus dem institutionellen Rahmen, auf Grund dessen Gesetze erlassen werden. ${ }^{7}$ Auch was der modale Charakter einer deontischen-normativen Aussage im Rahmen eines Erlasses faktisch beinhaltet, ist adäquat nur innerhalb des institutionellen Rahmens zu beschreiben, in dem Erlasse zu vollziehen sind. In der Normenlogik im Rahmen einer Möglichen-Welten-Semantik wird etwa eine deontische Aussage als Aussage über einen Sachverhalt definiert, die für eine besondere, "deontisch perfekte“ Welt gilt. ${ }^{8}$ In der Institution "Recht" hat aber natürlich der normative Charakter einer Gesetzesbestimmung konkretere Implikationen: Als Vorschrift steuert sie das Verhalten aller Beteiligten; für die Adressatinnen und Adressaten steuert sie Erwartungen darüber, was aus dem Nichtbefolgen eines Verbots oder Gebots folgt, für die staatlichen Institutionen, die mit dem Vollzug und der Rechtsdurchsetzung beauftragt sind, folgen daraus Handlungsverpflichtungen. Kurz: Der normative Sinn aus einer Bestimmung ist letztlich in den Auswirkungen auf das Handeln der unterschiedlich betroffenen Akteure zu beschreiben.

\footnotetext{
${ }^{6}$ Das Gegenstück sind, in der Terminologie des Schweizer Bundesrechts, Verfügungen, d. h. Anordnungen einer Behörde, die sich an eine konkrete Person in einem konkreten Sachverhalt richtet, beispielsweise als Aufforderung an eine Einzelperson, einen bestimmten Betrag als Steuer zu bezahlen.

${ }^{7}$ Aus welchen gesellschaftlichen oder politischen Gründen von einer Institution wie dem Staat überhaupt Gesetze erlassen werden und wie die Regelung durch ein Gesetz inhaltlich konzipiert werden soll, ist eine weitere Frage mit vielfältigen Antworten, die textuell-redaktionell nicht zu beantworten ist; siehe die Überblicksdiskussion bei GEORG MÜLLER, Elemente einer Rechtsetzungslehre. Zürich 1999, 9 ff.

${ }^{8}$ Zur Interpretation deontischer Aussagen innerhalb einer Möglichen-Welten-Semantik, worin die Bedeutung einer deontischen Aussage deren (wahrheitswertfunktionale) Geltung in einer entsprechenden deontisch perfekten Welt ist, siehe etwa GEORG KAMP, Logik und Deontik. Paderborn 2001, 235.
} 
Die institutionellen Voraussetzungen und Konsequenzen des normativen Charakters von Gesetzesbestimmungen scheinen zunächst direkt wenig mit der Textgrammatik von Gesetzen zu tun zu haben. Es hängen damit allerdings ganz konkrete Fragen der Gesetzgebung zusammen, wie die Frage der Zuständigkeit für eine Regelung oder jene der „Regelungsdichte“: Wer darf, soll oder muss wie viele Einzelheiten zu einem bestimmten Bereich regeln? Welches sind relevante Regelungsinhalte? Wo ist es überhaupt sinnvoll zu regeln, welche Bestimmungen sind notwendig oder überflüssig? Wer setzt eine Bestimmung in der Praxis um? Was sind die Folgen, wenn jemand eine Bestimmung nicht einhält? Und solche Fragen haben wiederum Auswirkungen auf die textuelle Gestaltung von Erlassen im Einzelnen.

\subsection{Allgemeine Vertextungsregeln für Erlasse}

Erlasse bestehen aus einer komplexen Menge von Bestimmungen. Welches ist das Grundprinzip, nach dem Bestimmungen zu möglichst kohärenten Texten vertextet werden können? Bei genauerer Betrachtung zeigt sich, dass aus normativen Bestimmungen aufgebaute Texte grundsätzlich den Vertextungsregeln von deskriptiven und narrativen Texten $^{9}$ folgen. Indem Bestimmungen Handlungen in Situationen vorschreiben oder verbieten oder Anforderungen an Gegenstände definieren, haben sie einen deskriptiven Gehalt: Sie beschreiben in der Regel bestimmte Verbindungen von situativen Voraussetzungen mit Handlungen oder Eigenschaften von Objekten, sie tun dies wie deskriptive Aussagen, lediglich unter dem besonderen modalen Aspekt des Gebots oder Verbots. So werden sie wohl auch, kognitiv gesehen, verstanden: Wer ein Gebot oder Verbot versteht, konstruiert sich ein kognitives Modell der Situation und der darin eingebetteten Handlung, wie sie der Gesetzgeber als vorgeschrieben oder untersagt definiert. Die zusätzliche modale „Färbung" des Verstehens besteht, wie oben erwähnt, darin, dass mit diesem speziellen Handlungsmodell, bestimmte praktische Implikationen allgemeiner oder besonderer Art verbunden werden. Das unmittelbare Verstehen einer Handlungsvorschrift wie der folgenden besteht also sachlich in der Rekonstruktion eines Handlungsmodells (bzw. eines Modells einer Teilhandlung), wonach man beim Inverkehrbringen von Stoffen gewisse begleitende Informationshandlungen vollzieht, und einer besonderen Bewertung dieses Handlungsmodells unter den möglichen davon abweichenden Alternativen:

\footnotetext{
${ }^{9}$ Vgl. zu dieser Unterscheidung MaRGOT HeINEMANN/WOLFGANG HEINEMANN, Grundlagen der Textlinguistik: Interaktion - Text - Diskurs. Tübingen 2002, $187 \mathrm{ff}$.
} 
${ }^{1}$ Wer Stoffe in Verkehr bringt, muss den Abnehmer:

a. über die umweltbezogenen Eigenschaften informieren;

b. so anweisen, dass beim vorschriftsgemässen Umgang mit den Stoffen die Umwelt oder mittelbar der Mensch nicht gefährdet werden kann.

(Umweltschutzgesetz, SR 814.01, Art. 17 Abs. 1) ${ }^{10}$

Wenn eine Regelung eine komplexe Folge von Handlungen regelt, bestehen zwischen den einzelnen Bestimmungen grundsätzlich dieselben thematischen Beziehungen, wie sie auch bei normalen deskriptiven Texten anzutreffen sind. Sie entstehen grundsätzlich durch entsprechende sachliche Beziehungen auf denotativer Ebene, auch dies analog zu deskriptiven und narrativen Texten. Wenn beispielsweise Bestimmungen einzelne Teilhandlungen eines größeren Handlungskomplexes regeln, so erscheinen diese Einzelbestimmungen durch diesen gemeinsamen sachlichen Bezug zu einer Gesamthandlung als zusammenhängend. Das Gleiche gilt für Vorschriften für Objekte oder Sachverhalte: Vorschriften für Teile einer Sache gehören auf Grund der denotativen Zusammengehörigkeit auch textuell zusammen. Ebenso schafft das Hyponymieverhältnis textuelle Zusammengehörigkeit zwischen allgemeineren Bestimmungen für eine Handlung oder eine Sache und Sonderbestimmungen wie Ausnahmebestimmungen oder Bestimmungen für besondere Teilaspekte oder Sondersituationen. Derartige thematische Beziehungen auf denotativer Grundlage zwischen den einzelnen Bestimmung wirken in Erlasstexten in gleicher Weise kohärenzstiftend wie bei Ereignis- oder Handlungsbeschreibungen. Und schließlich kann man in solchen Fällen auch die geläufigen Linearisierungsprinzipien anwenden, etwa, dass das Allgemeine vor dem Besonderen genannt werden soll oder dass Beschreibungen von Handlungsabläufen ikonisch entsprechend der chronologischen Abfolge geordnet werden sollen.

Als Beispiel können etwa die Artikel 16 bis 20 der Agrareinfuhrverordnung angeführt werden, in denen das Verfahren geregelt wird, wie Zollkontingente für die zollbegünstigte Einfuhr landwirtschaftlicher

\footnotetext{
${ }^{10}$ Die Fundstelle von Erlassen des Schweizerischen Bundesrechts wird mit Verweis auf die entsprechende Ziffer in der Systematischen Rechtssammlung (SR) angegeben; es handelt sich dabei stets um die aktuelle konsolidierte Fassung. Die SR ist über das Internet unter der URL http://www.admin.ch/ch/d/sr/sr.html allgemein zugänglich.
} 
Produkte zu versteigern sind. Darin sind - im Wesentlichen chronologisch - der Reihe nach Bestimmungen enthalten über die Ausschreibung, die Eingabe für Steigerungsgebote, die Regeln der Zuteilung, die Bestimmung des Zuschlagspreises und die Zahlungsfrist. In einzelnen Artikeln werden dem noch einzelne Sonderbestimmungen hinzugefügt: In Artikel 17 Absatz 2 etwa wird die Zahl der erlaubten Gebote pro bietender Person und die Fristen für Änderungen oder Rückzüge von Geboten eingeschränkt. In Artikel 18 Absätze 2 und 3 werden bestimmte Sondersituationen geregelt, für die die allgemeinen Regeln in Absatz $1 \mathrm{zu}$ vage sind. Diese Sonderbestimmungen beziehen sich auf Teilaspekte, die implikativ als mögliche Teilsituation innerhalb der Möglichkeiten im gesamten geregelten Handlungskomplex gegeben sind.

\section{Abschnitt: Versteigerung}

\section{Art. 16 Ausschreibung}

Das Bundesamt schreibt die Versteigerung im Schweizerischen Handelsamtsblatt aus.

\section{Art. 17 Steigerungsgebote}

${ }^{1}$ Die Steigerungsgebote sind dem Bundesamt auf dem dafür vorgesehenen Formular innert der in der Ausschreibung festgesetzten Frist einzureichen.

${ }^{2}$ Jede bietende Person kann für die ausgeschriebene Menge maximal fünf Gebote mit verschiedenen Preisen und Mengen einreichen.

${ }^{3}$ Die Gebote können nach Ablauf der Einreichungsfrist weder geändert noch zurückgezogen werden.

\section{Art. 18 Zuteilung}

${ }^{1}$ Die Zuteilung erfolgt, beginnend beim höchsten gebotenen Preis, in abnehmender Reihenfolge der gebotenen Preise.

${ }^{2}$ Auf dem tiefsten noch zu berücksichtigenden Preisniveau wird gegebenenfalls eine proportional gekürzte Menge zugeteilt. Falls die zugeteilte Menge kleiner ist als die Mindesteingabemenge, kann die bietende Person ihr Gebot zurückziehen.

${ }^{3}$ Wird durch die Zuteilung die ausgeschriebene Zollkontingentsmenge nicht voll ausgenützt, so kann die Restmenge: 
a. unter die erstmalig bietenden Personen auf dem Zirkularweg neu ausgeschrieben werden; oder

b. nochmals allgemein ausgeschrieben werden.

Art. 19 Zuschlagspreis und Zahlungsfrist

${ }^{1}$ Der Zuschlagspreis entspricht dem Gebotspreis.

${ }^{2}$ Die Einfuhr zum Kontingentszollansatz (KZA) oder, bei Einfuhren im Rahmen der Zollkontingente 119-123 nach Anhang 2 der Verordnung vom 8. März 2002 über die Ein- und Ausfuhr von Käse zwischen der Schweiz und der Europäischen Gemeinschaft, zum Nullzoll ist erst zulässig, wenn der gesamte Zuschlagspreis bezahlt worden ist.

${ }^{3}$ Die Zahlungsfrist beträgt, vorbehältlich von Absatz 2, 60 Tage nach Rechtskraft des Zuschlags.

${ }^{4}$ Die Einfuhr zum Kontingentszollansatz (KZA) oder zum Nullzoll ist auch zulässig, wenn dem Bundesamt vor der Einfuhr eine Bankgarantie oder andere, nach Artikel 43 der Finanzhaushaltverordnung vom 11. Juni 1990 gestattete Garantie zugestellt worden ist. Die Sicherstellung muss dem Zuschlagspreis entsprechen.

${ }^{5}$ Ausnahmen sind in den marktordnungsspezifischen Produkteverordnungen geregelt.

Art. 20 Veröffentlichung der Zuteilung

Die Zuteilung der Zollkontingentsanteile wird im Schweizerischen Handelsamtsblatt veröffentlicht.

(Agrareinfuhrverordnung, SR 916.01)

\section{Normative Relevanz}

\subsection{Lex iubeat, non doceat}

Wenn für Erlasse generell nur die beschriebenen Grundprinzipien der Vertextung deskriptiver Texttypen gelten würden, könnten sie als Texte so verständlich sein wie sonstige deskriptive Texttypen. Jedoch gelten für Erlasse zusätzlich einige besondere Grundregeln, die sie von anderen Texttypen unterscheiden. Ein erstes Grundprinzip lautet: Ein Erlass darf nur normativ relevante Elemente enthalten, d. h. Elemente, 
die wirklich etwas gebieten, verbieten usw. Eine alte Maxime für die Formulierung von Gesetzen lautet "Lex iubeat, non doceat"11: Das Gesetz soll befehlen, nicht lehren. Dass ein normativer Text wie ein Erlass nur Vorschriften enthalten soll, scheint zunächst auf Grund von dessen genereller Zweckbestimmung nur natürlich. Die Maxime ist jedoch präziser gemeint; sie wird gemeinhin so verstanden, dass ein Gesetzestext überhaupt nur normative Aussagen bzw. umgekehrt: keine nichtnormativen Aussagen enthalten soll wie etwa metakommunikative Erläuterungen, Informationen zu Hintergrundwissen oder Zweckbegründungen. Gesetzestexte sind grundsätzlich "monofunktional", nicht nur im Hinblick auf ihren illokutiven Gehalt im Ganzen, sondern auch auf den Gehalt der einzelnen Teile. Dies unterscheidet sie von anderen Texttypen, in denen inhaltlich und sprachlich Mischungen und Kombinationen von verschiedenen textuellen Elementtypen gang und gäbe sind.

Das Verbot nichtnormativer Elemente in Erlassen hat eine alte Tradition, wie schon die lateinische Maxime erkennen lässt. Es lässt sich aus der besonderen Funktion von Gesetzen begründen: Gesetze sollen überindividuell und generell Regelungen festlegen, unabhängig von der Meinung einzelner Personen. Erwägungen, Begründungen und Diskussionen, die zum Erlass einer Bestimmung geführt haben oder ihren Zweck begründen, sind gewöhnlich situationsbezogen, individuell, und unter Umständen gelten sie sogar nicht einhellig, selbst wenn sich alle an der Gesetzgebung Beteiligten über eine bestimmte Formulierung für eine bestimmte Regelung geeinigt haben. Hintergrundwissen ist kontingent und adressatenabhängig. Auch würden Selbstkommentierungen von Gesetzesaussagen dem institutionellen, überindividuellen Charakter von Gesetzen widersprechen. Die unterschiedlichen Textfunktionen müssen im Bereich des Rechts scharf in verschiedene Textsorten getrennt werden, damit die Autorität des Rechts gewahrt bleibt. Das Verbot autorenspezifischer Zusätze ist ferner eine wesentliche Garantie der Eindeutigkeit und Sicherheit der Interpretation. Bestünde das Verbot nicht, wäre bei sehr vielen Arten von Elementen nicht entscheidbar, ob sie als Teil einer Norm zu verstehen sind oder lediglich als normativ irrelevante Zusätze, und bei der Auslegung wäre der Willkür Tür und Tor geöffnet. Überdies könnten Erläuterungen und Begründungen selbst Stoff der Auslegung werden und damit auch die Auslegung der eigentlichen normativen Teile von Gesetzen verwirren.

\footnotetext{
11 Varianten lauten: "Lex moneat, non doceat", "Lex iubeat, non disputet" (Das Gesetz soll befehlen, nicht argumentieren).
} 


\subsection{Regelungsdichte und Deskriptivität}

Das Prinzip der normativen Relevanz hat weit reichende Auswirkungen auf die Gestaltung von Erlasstexten. Von gewöhnlichen, prototypischen kohärenten deskriptiven Texten erwartet man eine gewisse angemessene Informativität und Vollständigkeit: Mindestens soviel Information sollte angeführt werden, wie notwendig ist, damit der Adressat/die Adressatin sich ein vollständiges Bild vom beschriebenen Sachverhalt machen kann. Der Umfang der notwendigen Information ist zwar vom Zweck eines Textes, vom Vorwissen des Adressaten/der Adressatin, vom intendierten Detaillierungsgrad u. a. m. abhängig. Die Beschreibung eines Handlungsablaufs sollte alle einzelnen Handlungsschritte im gleichen Detaillierungsgrad darstellen sowie eventuelle relevante Abweichungen vom normalen zu erwartenden Handlungsverlauf oder von normalerweise gemachten impliziten Voraussetzungen erwähnen. Ebenso sollte die Beschreibung eines Objekts alle Teile und Aspekte etwa in gleicher Beschreibungsdichte darstellen. Für normative Texte ist demgegenüber auf Grund des Prinzips der normativen Relevanz die angemessene Informativität in diesem Sinn kein maßgebendes Qualitätskriterium.

Erlasse haben von ihrer Funktion her nicht den Zweck, ein angemessen detailliertes mentales Modell konstruieren $\mathrm{zu}$ helfen und müssen keine abgeschlossene kohärente Beschreibung eines Realitätsausschnitts als Komplex von Sachverhalten liefern. Vielmehr soll ein Erlass nur solche Aspekte eines Handlungsmodells oder eines Sachverhaltsausschnitts regeln, deren Regelung auf Grund der gesetzlichen Vorgaben, aus dem Zweck des Erlasses, aus der Sache und von der Praktikabilität der Umsetzung her als erforderlich erscheint. Alle jene Details eines Handlungsmusters, deren Realisierung dem Ermessen der direkt Betroffenen anheim gestellt werden kann, sind demzufolge offen zu lassen. Ein Gesetz hat entsprechend oft mehr einen "Checklistenaufbau": Nur jene Punkte werden behandelt, die als regelungsbedürftig beachtet werden müssen. Die Kenntnis eines Handlungsablaufs oder eines Sachverhalts wird vorausgesetzt und nicht vermittelt. Im folgenden Beispiel wird der Nationalbank die Kompetenz erteilt, dem Bund Bankdienstleistungen zu erbringen. Was Bankdienstleistungen sind, wird nicht erläutert; das Wissen über die üblichen Dienstleistungen wird vorausgesetzt. Im zweiten Absatz wird lediglich für einzelne dieser Bankdienstleistungen ausgeführt, dass sie untersagt sind; wie es sich mit den Übrigen verhält, muss aus dem Nichterwähnen erschlossen werden. 
Art. 11 Geschäfte für den Bund

${ }^{1}$ Die Nationalbank kann dem Bund Bankdienstleistungen erbringen. Sie erbringt diese gegen angemessenes Entgelt, jedoch unentgeltlich, wenn sie die Durchführung der Geld- und Währungspolitik erleichtern. Die Einzelheiten werden in Vereinbarungen zwischen Bundesstellen und der Nationalbank geregelt.

${ }^{2}$ Die Nationalbank darf dem Bund weder Kredite noch Überziehungsfazilitäten gewähren; sie darf auch nicht staatliche Schuldtitel aus Emissionen erwerben. Sie kann Kontoüberziehungen im Verlaufe des Tages gegen ausreichende Sicherheiten zulassen.

(Bundesgesetz über die Schweizerische Nationalbank, SR 951.11)

Diese Selektivität wirkt für die an normale Sachtexte gewohnten Leser und Leserinnen eher verwirrend. Es fehlt oft der "rote Faden". Erst recht gilt das für den Aufbau eines umfänglicheren Gesetzes. Hier wirkt sich die deskriptive Selektivität oft so aus, dass die Teile nur locker oder gar nicht miteinander verbunden sind; sie erscheinen nur deshalb als zusammengehörig, weil sie eine übergreifende Regelungsthematik behandeln. Die Kapiteleinteilung des Nationalbankgesetzes vom 3. Oktober 2003 etwa zeigt ein Mosaik ziemlich heterogener Regelungsinhalte:

Kapiteleinteilung des Bundesgesetzes über die Schweizerische Nationalbank

1. Kapitel: Allgemeine Bestimmungen

2. Kapitel: Geschäftskreis (= erlaubte Arten von Bankgeschäften)

3. Kapitel: Geld- und währungspolitische Befugnisse

4. Kapitel: Aktienrechtliche Bestimmungen

5. Kapitel: Organisation

6. Kapitel: Verfahren und Rechtsschutz

Besonders heterogen sind die in Kapitel 1 eingereihten Artikel, die u. a. die Rechtsform, die Schaffung von Zweigniederlassungen, das Notenmonopol, den Grundsatz der Unabhängigkeit und die Steuerbefreiung regeln.

Die Teile von Erlassen sind also thematisch oft kaum miteinander verbunden. Solche Erlasse besitzen deshalb nur eine schwache Kohärenz zwischen ihren Teilen. 


\subsection{Normative Relevanz und verständnisunterstützende Information}

Texte $z u$ verstehen impliziert mehr als nur die einfache Interpretation der direkten Informationen in einem Text über einen Sachverhalt, eine Handlung oder eine Argumentation. Wichtig ist auch, dass der Rezipient/die Rezipientin übergreifende Zusammenhänge zwischen den Handlungen versteht, auch Begründungen und Informationen über Motivationen zu Handlungen erkennt, das Ganze in einen weiteren Zusammenhang stellen kann, Hintergrundwissen darüber erhält, warum eine bestimmte Information relevant ist $(\mathrm{d} . \mathrm{h}$. worin das Neue in einer Aussage besteht). Falls dies nicht ohne weiteres aus dem Text erschließbar ist, hat ein Text unter Umständen auch Informationen zu enthalten, die streng genommen nicht zum Thema eines Textes (zu dem, was der Text als relevante Information übermitteln soll) gehören.

Auch in diesem Punkt setzt das Prinzip der normativen Relevanz der Formulierung von Erlassen enge Schranken: Alle Ergänzungen wie Kommentierung, Begründung, Erläuterung oder auch der metakommunikativen Verknüpfung von Aussagen sind bei der Formulierung von Gesetzesbestimmungen ausgeschlossen. Solche Mittel sind es aber gerade, welche in Alltagstexten sich häufig als probate Verfahren zum Verständlichmachen von Texten anbieten. Das Resultat des Verbots solcher Aussagen ist, dass Gesetze aus unerklärten und unerläuterten Aneinanderreihungen von Aussagen bestehen. Auch wird fast immer auf textverknüpfende Partikeln und Formeln wie "demgegenüber", „im Gegensatz dazu“, „darüber hinaus" verzichtet, welche die Zusammenhänge verdeutlichen. Diese textlinguistische Abstinenz ist eines der auffälligsten verständnishemmenden Merkmale von Gesetzestexten. Um etwa zu erkennen, welches das logische Verhältnis zweier aufeinanderfolgender Bestimmungen zueinander ist, ob eine Aussage z. B. eine Ausnahmebestimmung zu einer anderen ist oder zwei gleichgeordnete Bestimmungen vorliegen, müssen die einzelnen Aussagen sehr genau interpretiert und die entsprechenden Schlussfolgerungen ohne Unterstützung durch textlinguistische Hilfen erschlossen werden. So wird z. B. im oben angeführten Beispiel der Versteigerung unkommentiert ein Versteigerungsverfahren geregelt, ohne dass beispielsweise erläutert wird, dass bei der Versteigerung ein bestimmtes unter mehreren möglichen Verfahren angewendet wird, nämlich das bei der Emission von Wertpapieren gebräuchliche Tenderverfahren.

Gesetzestexte unterstützen einen allfälligen Bedarf an Hintergrundwissen und Erklärungen zu einzelnen Bestimmungen nicht und verletzen damit oft in eklatanter Weise das von Alltagstexten her gewohnte und erwartete Kooperativitätsprinzip. 


\subsection{Erläuterungen und Kommentare}

Wenn oben gesagt wurde, dass Erlasse keine Erläuterungen und Begründungen als Textelemente enthalten, dann heißt das nicht, dass es solche Informationen zu Erlassen nicht gibt. Im Gegenteil: Es gibt bestimmte Textsorten, die zum Teil in verbindlicher Form diesen Zweck erfüllen. Erläuterungen finden sich in der Schweizer Gesetzgebung namentlich als Begleitdokumente zu einer Vorlage an die erlassende Institution. Gesetzlich geregelt ist, dass zu jedem Gesetzesentwurf, der vom Bundesrat dem Parlament zur Beratung und Verabschiedung unterbreitet wird, ein erläuternder und begründender Text, eine so genannte "Botschaft" gehört. Auf diese Botschaft wird auch im Ingress zu einem Gesetz verwiesen. Die Botschaft und ihr Inhalt ist auch ein maßgebendes Dokument für die Beratung durch das Parlament und die spätere Anwendung des Gesetzes durch Behörden und Gerichte. Auch bei Verordnungen unterer Stufe werden je nach Bedarf Erläuterungen verfasst.

$\mathrm{Zu}$ wichtigen Gesetzen mit ihren Verordnungen gibt es schließlich noch weitere Begleitdokumente, welche den Inhalt von gesetzlichen Bestimmungen zusätzlich präzisieren, nämlich Kommentare und Gerichtsurteile mit ihren Begründungen. Kommentare präzisieren und erläutern den Inhalt der verschiedenen Bestimmungen durch Zusammenstellung der Zusatzinformationen aus behördlichen Erläuterungen, parlamentarischen Beratungen, Verknüpfung mit anderen gesetzlichen Bestimmungen und gerichtlichen Erwägungen. Gerichtsurteile können einzelne Bestimmungen auch normativ näher begrenzen und so Unklarheiten, Grenz- und Zweifelsfälle durch zusätzliche Festlegungen klären.

Die Existenz dieser Zusatzdokumente ist eine zusätzliche Konsequenz und ein weiterer Ausdruck der Regel, dass im Recht Inhalte von unterschiedlicher Funktion und mit unterschiedlichen pragmatischen Zwecken auch in unterschiedlichen Textsorten mit unterschiedlichen Textsortenregeln gefasst werden. Umso reiner kann in Erlassen der Grundsatz der normativen Relevanz realisiert werden. Andererseits muss, wer den vollen Gehalt einer Gesetzesbestimmung erkennen will, oft mehrere Texte berücksichtigen. 


\section{Identität von Regelung und Bestimmung}

\subsection{Identitätsprinzip}

Ein weiterer wichtiger Grundsatz in der Gesetzesredaktion lautet: Die Regelung muss mit ihrer textuellen Realisierung identisch sein. Anders gesagt: Eine bestimmte Regelung muss sich in einer textuell eindeutig fassbaren Bestimmung finden, und umgekehrt: Eine Bestimmung hält eindeutig eine bestimmte Regelung fest. Von Gesetzesredaktoren und -redaktorinnen wird dieses Prinzip wohl selten explizit reflektiert; es wird in seinen Konsequenzen aber im Allgemeinen streng beachtet und wirkt sich in der ganzen Ausgestaltung von Gesetzen nachhaltig aus. Textlich konkretisiert sich dieses Prinzip beispielsweise in der Maxime "Ein Satz - eine Aussage"; d. h. in einem Satz soll nur eine minimale Bestimmung enthalten sein. ${ }^{12}$ Eine andere Konsequenz ist das Wiederholungsverbot (siehe dazu den nächsten Abschnitt).

Der Grundsatz der Identität von Regelung und Bestimmung lässt sich mehrfach begründen. Ein sprachtheoretischer Grund liegt darin, dass eine Regelung textuell eindeutig identifizierbar sein muss, damit sie eindeutig auslegbar und anwendbar ist. Andernfalls wäre nicht erkennbar, ob zwei parallele Bestimmungen mehreren Regelungen entsprechen oder nicht. Wäre es möglich, dass in einer Bestimmung mehrere Regelungen enthalten sind oder eine Regelung auf verschiedene Bestimmungen verteilt ist, wäre nicht präzis und klar entscheidbar, wie viele Regelungen in einer bestimmten Hinsicht auf einen konkreten Sachverhalt anzuwenden wären. Praktisch würde schließlich die Wiederholung einer Regelung in mehreren Bestimmungen zu Problemen bei jeder Änderung eines Erlasses führen: Es bestünde die Gefahr, dass eine Bestimmung nur an einem Ort geändert würde, wodurch schließlich mehrere widersprüchliche Regelungen zu ein und demselben Sachbereich gelten würden.

\subsection{Wiederholungsverbot}

Eine wichtige Implikation des Identitätsprinzips besteht im Verbot, eine gleiche Norm an zwei Orten aufzuführen, sei es im gleichen Erlass,

\footnotetext{
12 Diese besondere Maxime ist nicht immer leicht durchzuhalten; oft ist auch nicht ganz klar, wie viele Regelungen in einem Satz oder in zwei Sätzen enthalten sind oder ob in einer Aufzählung mehrere Regelungen oder eine extensionale Charakterisierung einer einzelnen Regelung vorliegt oder ob eine adverbiale Erweiterung eine zusätzliche Regelung oder eine Präzisierung einer Regelung bedeutet.
} 
sei es in verschiedenen Erlassen. Das Wiederholungsverbot ist ebenfalls ein Grundsatz, der Erlasstexte von normalen Typen von Sachtexten unterscheidet: In letzteren besteht überhaupt kein Hindernis, bestimmte Aussagen bei Bedarf zu wiederholen, außer allenfalls in der allgemeinen Maxime, der Adressat/die Adressatin solle nicht mit Redundanz belästigt werden. Das Wiederholungsverbot für Erlasstexte erschwert allerdings in vielen Fällen eine direkt verständliche Formulierung von Regelungen, vor allem dann, wenn eine einzelne Bestimmung ein engeres inhaltliches Verhältnis zu mehreren anderen Bestimmungen besitzt, $z$. B. wenn eine Bestimmung eine Voraussetzung oder eine Rahmenbedingung anderer Bestimmungen bildet. So werden inhaltliche Zusammenhänge innerhalb eines Erlasses auseinander gerissen und erst bei eingehender Lektüre des ganzen Erlasses erkennbar. Noch schwerwiegender sind die Verständnisprobleme, wenn Regelungen in zwei verschiedenen Erlassen aufeinander bezogen sind. Im folgenden Artikel aus dem Nationalbankgesetz wird beispielsweise auf "das Aktienbuch" (mit bestimmtem Artikel!) verwiesen, ohne dass der Begriff eingeführt und dessen Funktion geregelt würde. Stillschweigend wird dabei eine entsprechende Regelung im Obligationenrecht vorausgesetzt, wo das Aktienbuch behandelt wird. (Da ein entsprechender erläuternder Verweis nur ein nichtnormativer Kommentar wäre, wird auch diese Verstehenshilfe nicht geliefert).

\section{Art. 26 Aktienbuch, Übertragungsbeschränkungen}

${ }^{1}$ Die Nationalbank anerkennt als Aktionärinnen und Aktionäre nur, wer im Aktienbuch eingetragen ist. Der Bankrat regelt die Einzelheiten der Eintragung.

2 Die Eintragung einer Aktionärin oder eines Aktionärs ist auf höchstens 100 Aktien beschränkt. Diese Beschränkung gilt nicht für schweizerische Körperschaften und Anstalten des öffentlichen Rechts sowie für Kantonalbanken im Sinne von Artikel $3 a$ des Bankengesetzes vom 8. November 1934.

${ }^{3}$ Die Eintragung wird verweigert, wenn die Erwerberin oder der Erwerber trotz Verlangen der Nationalbank nicht ausdrücklich erklärt, die Aktien im eigenen Namen und auf eigene Rechnung erworben zu haben und zu halten.

(Bundesgesetz über die Schweizerische Nationalbank, SR 951.11) 


\subsection{Isolierung der Einzelbestimmung}

Gesetze weisen in der Darstellung als nummerierte Artikel und Absätze, die je in sich geschlossene Einheiten sind, eine Textgestaltung auf, die von derjenigen gewöhnlicher Sachprosa als Fließtext markant abweicht. Diese Darstellung hat sich in einer Jahrhunderte alten Tradition herausgebildet. Sie dürfte in der tief sitzenden Überzeugung begründet sein, wonach jede minimale Regelung eine für sich geltende Regelung ist, die als solche erkennbar sein muss und unabhängig von anderen Regelungen ihr eigenes Gewicht hat. Dieses Prinzip ist mit dem Prinzip der Identität von Regelung und Bestimmung eng verwandt. Jede Bestimmung muss für sich einzeln anwendbar sein, für jede einzelne Bestimmung muss die Anwendung separat überprüft werden können. In der Praxis gilt die Maxime, dass jeder Absatz für sich als selbstständige Aussage auch außerhalb seines Kontextes zitierbar sein muss. Die isolierende Darstellung und Formulierung widerspiegelt außerdem das Prinzip, dass jede einzelne Bestimmung in der Anwendung gleich wichtig ist, auch wenn eine Bestimmung eine andere voraussetzen mag. Eine Detailbestimmung kann für Rechtsbetroffene größere Auswirkungen haben als eine allgemein gehaltene Generalklausel. Ein Erlass lässt sich deshalb auch nicht wie ein deskriptiver oder argumentativer Text in einem präzisen Sinn so vereinfachen und zusammenfassen, dass der Sinn des Erlasses in einem genauen Sinn erhalten bliebe. ${ }^{13}$

Zusammen mit der Regel, dass metasprachliche Erläuterungen und Übersichten nicht erlaubt sind, führt die isolierende Darstellung von Bestimmungen dazu, dass Zusammenhänge zwischen einzelnen Bestimmungen sprachlich oft nur indirekt erkennbar sind. Als Beispiel sei der Abschnitt über die Abfallbehandlung im Umweltschutzgesetz erwähnt:

Art. $30 \quad$ Grundsätze
${ }^{1}$ Die Erzeugung von Abfällen soll soweit möglich vermieden werden.
${ }^{2}$ Abfälle müssen soweit möglich verwertet werden.
${ }^{3}$ Abfälle müssen umweltverträglich und, soweit es möglich und sinn-
voll ist, im Inland entsorgt werden.

${ }^{13}$ Vom rechtlich-materiellen Standpunkt aus kann man in Gesetzen also nicht Wichtigeres von Unwichtigerem unterscheiden, wie das in gewöhnlichen Sachtexten der Fall ist. Allenfalls lassen sich generellere von spezielleren Regelungen unterscheiden. 
Art. 30a Vermeidung

Der Bundesrat kann:

a. das Inverkehrbringen von Produkten verbieten, die für eine einmalige und kurzfristige Verwendung bestimmt sind, wenn deren Nutzen die durch sie verursachte Umweltbelastung nicht rechtfertigt;

b. die Verwendung von Stoffen oder Organismen verbieten, welche die Entsorgung erheblich erschweren oder bei ihrer Entsorgung die Umwelt gefährden können;

c. Hersteller verpflichten, Produktionsabfälle zu vermeiden, für deren umweltverträgliche Entsorgung keine Verfahren bekannt sind.

\section{Art. $30 \mathrm{~b}$ Sammlung}

${ }^{1}$ Der Bundesrat kann für bestimmte Abfälle, die zur Verwertung geeignet sind oder besonders behandelt werden müssen, vorschreiben, dass sie getrennt zur Entsorgung übergeben werden müssen.

${ }^{2}$ Er kann denjenigen, die Produkte in Verkehr bringen, welche als Abfälle zur Verwertung geeignet sind oder besonders behandelt werden müssen, vorschreiben:

a. diese Produkte nach Gebrauch zurückzunehmen;

b. ein Mindestpfand zu erheben und dieses bei der Rücknahme zurückzuerstatten.

${ }^{3}$ Er kann für die Schaffung einer Pfandausgleichskasse sorgen und insbesondere vorschreiben, dass:

a. diejenigen, die pfandbelastete Produkte in Verkehr bringen, Überschüsse aus der Pfanderhebung der Kasse abliefern müssen;

b. die Überschüsse für die Deckung von Verlusten aus der Pfandrückerstattung und für die Förderung des Rücklaufs pfandbelasteter Produkte verwendet werden müssen.

Art. 30c Behandlung

${ }^{1}$ Abfälle müssen für die Ablagerung so behandelt werden, dass sie möglichst wenig organisch gebundenen Kohlenstoff enthalten und möglichst wasserunlöslich sind.

${ }^{2}$ Abfälle dürfen ausserhalb von Anlagen nicht verbrannt werden; ausgenommen ist das Verbrennen natürlicher Wald-, Feld- und Gartenabfälle, wenn dadurch keine übermässigen Immissionen entstehen. 
${ }^{3}$ Der Bundesrat kann für bestimmte Abfälle weitere Vorschriften über die Behandlung erlassen.

(Umweltschutzgesetz, SR 814.01)

Der ganze Abschnitt formuliert in Regelungsform sinngemäß das Prinzip, dass Abfälle primär vermieden, wenn dies nicht möglich ist, verwertet, wenn dies nicht möglich ist, umweltfreundlich entsorgt werden sollen. Diese drei Stufen werden auch in Artikel 30 der Reihe nach genannt, aber als isolierte, nicht aufeinander bezogene Bestimmungen. Dass in der Aufzählung eine Priorisierung enthalten ist, muss man aus der Reihenfolge sozusagen als ikonisch vorhandene Information erschließen. Zu den einzelnen Verfahrensweisen folgen anschließend in detaillierteren Bestimmungen noch nähere Regelungen, aber auch hier nicht unter explizitem Verweis auf die Grundsätze, sondern nur durch die jeweilige Erwähnung der Schlüsselwörter. Dadurch erkennt man den Zusammenhang der Detailbestimmungen mit den Grundsätzen nur durch Verknüpfung der Aussagen beim mehrmaligen Lesen.

Im Übrigen ergibt sich aus der Regel, dass ein Absatz isoliert zitierbar sein muss, auch die Konsequenz, dass anaphorische Bezüge zwischen Absätzen und Artikeln nicht erlaubt sind. Sie werden höchstens innerhalb einzelner Absätze toleriert. Aus dem Kontext gerissen sind ja deiktische und anaphorische Bezüge nicht mehr rekonstruierbar. Dies zwingt vielfach zu umständlichen Verweisen wie "das Gesuch nach Absatz/nach Artikel 10", die in sich auch informationsarm sind.

\section{Prinzip der funktionalen Gruppierung}

Ein weiteres Phänomen der Textorganisation von Erlassen könnte man als "Prinzip der funktionalen Gruppierung” bezeichnen: In vielen Fällen werden Bestimmungen, die sachlich zusammengehören, aber unterschiedliche Regelungstypen repräsentieren, auseinander genommen und nach Regelungstyp gruppiert. Geläufig ist das Verfahren, sämtliche Legaldefinitionen am Anfang eines Erlasses zusammenzufassen. Dies ist textorganisatorisch sinnvoll, wenn es sich um Legaldefinitionen von Begriffen handelt, die im ganzen Erlass zentral sind und häufig wiederkehren. In solche Begriffslisten werden dann aber auch Definitionen von Begriffen aufgenommen, die nur ein oder zweimal an versteckter Stelle vorkommen. Der Leser oder die Leserin ist so gezwungen, bei allen potenziellen Zweifelsfällen im entsprechenden Katalog der Begriffsbestimmungen nachzusehen, ob ein problematischer Aus- 
druck definiert ist. In einer Vielzahl von Erlassen finden sich am Anfang auch "Allgemeine Bestimmungen", die für alle im Nachfolgenden behandelten Einzelfälle gemeinsam gelten. Weitere Gruppen von Bestimmungen, die konventionellerweise zusammen gruppiert werden, allerdings am Ende eines Erlasses, sind Verfahrensbestimmungen, Strafbestimmungen und Übergangsbestimmungen. Wer also wissen will, wie das Verfahren bei Rechtshandlungen und die Strafe für Zuwiderhandlungen gegen eine Bestimmung geregelt sind, oder ob für eine bestimmte Regelung eine Übergangsbestimmung gilt, muss sich an einem anderen Ort informieren, als dort, wo die sachlichen Bestimmungen selbst eingeordnet sind.

Für derartige Gruppierungen gibt es unterschiedliche Gründe. Dass beispielsweise Übergangsrecht am Schluss eines Erlasses geregelt wird, ist erklärlich aus dem Umstand, dass es sich um „vergängliche“ Bestimmungen handelt, die nach einiger Zeit obsolet werden; "echte" "vollwertige" Gesetzesbestimmungen gelten demgegenüber sozusagen zeitlos. Von der zeitlichen Natur der Geltung her ist es sinnvoll, solche unterschiedlichen Regelungstypen zu trennen. In anderen Fällen, wie bei Verfahrensbestimmungen und Strafbestimmungen, schafft die $\mathrm{Zu}-$ sammenfassung an einem einzigen Ort für bestimmte Adressaten, etwa Verwaltungsbehörden oder gerichtliche Instanzen, die von einzelnen Regelungstypen besonders betroffen sind, auch mehr Übersichtlichkeit. Generell sind es aber wohl nicht derartige subtile Überlegungen, welche die Gesetzesredaktoren und -redaktorinnen dazu gebracht haben, Gesetzesbestimmungen nach Regelungstypen zu trennen, sondern die Anwendung des Prinzips "Gleiches zu Gleichem" auch in funktionaler Hinsicht. Daraus haben sich Gewohnheiten mehr konventioneller Art ergeben.

\section{Intertextualität}

\section{1 Kompetenzdelegationen und Kollisionsnormen}

Nicht immer wird in einem Erlass ein Sachbereich direkt und vollständig geregelt. Vielmehr kann die Regelungskompetenz an eine untergeordnete Behörde delegiert werden (Delegationsnorm) oder es wird auf einen anderen Erlass oder eine andere Bestimmung im gleichen Erlass verwiesen, in dem zum gleichen Sachbereich oder einem Teilbereich ebenfalls Regelungen enthalten sind; damit wird das Verhältnis der beiden Erlasse zueinander geregelt. Ein Beispiel bilden etwa die Artikel 29 a und $29 \mathrm{~b}$ aus dem Umweltschutzgesetz: 


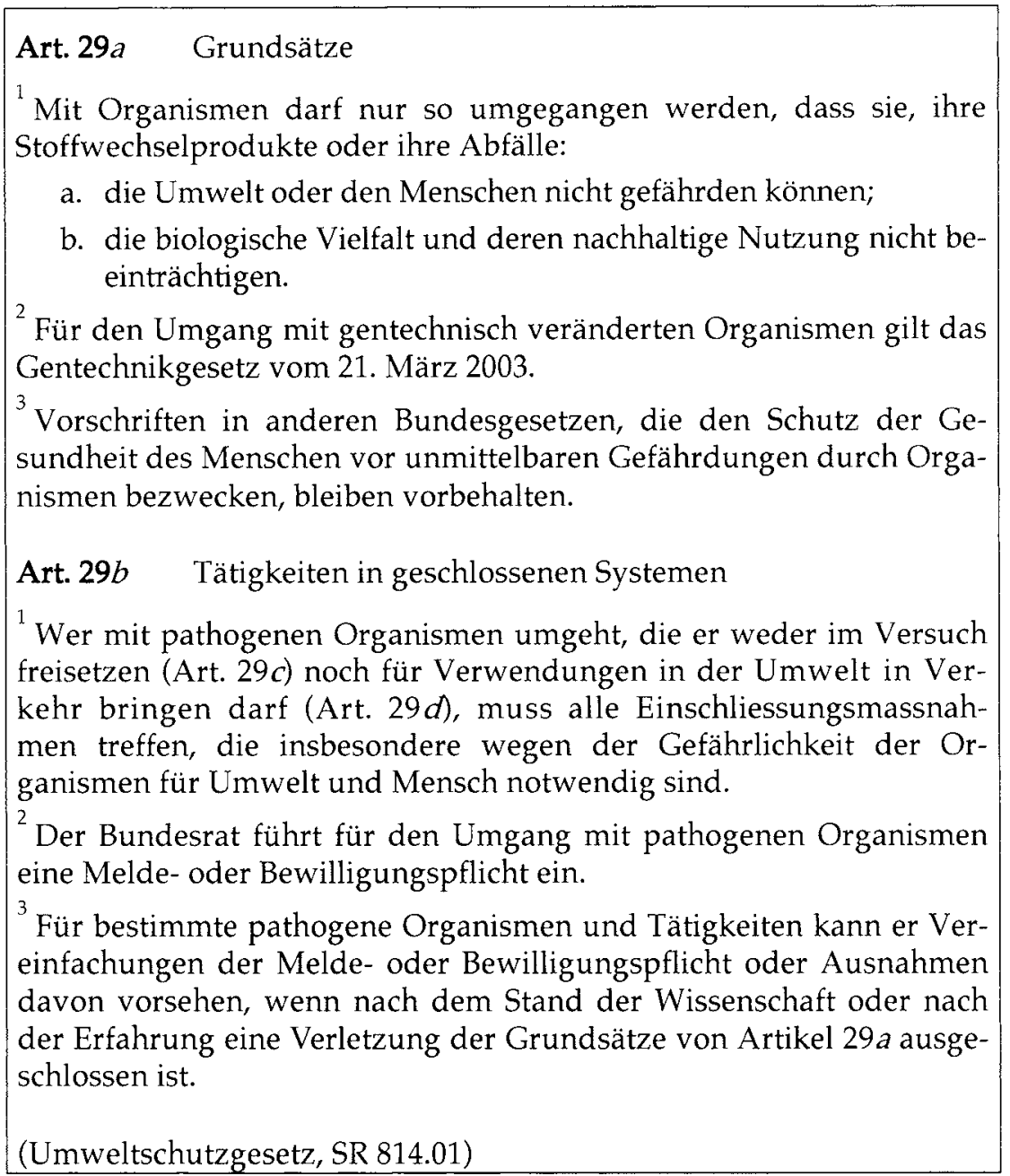

Artikel 29a regelt allgemeine Grundsätze zum Umgang mit Organismen; in Artikel 29a Absatz 2 wird auf Sonderbestimmungen für gentechnisch veränderte Organismen in einem anderen Gesetz verwiesen, in Absatz 3 auf generelle Bestimmungen zum Gesundheitsschutz. Der Sinn solcher Bestimmungen ist, das gegenseitige Verhältnis der verschiedenen Bestimmungen zueinander zu präzisieren. In Artikel $29 \mathrm{~b}$ wird in den Absätzen 2 und 3 dem Bundesrat eine Regelungskompetenz übertragen. Textgrammatisch hat die Einfügung von Verweisnormen und Delegationsnormen zur Folge, dass die Leserin/der Leser an 
der betreffenden Stelle nicht alle relevanten Bestimmungen inhaltich ausgeführt antreffen kann, sondern auf andere Erlasse verwiesen wird.

Soweit die einzelnen Bestimmungen in einem Erlass sich thematisch auf Teile eines komplexen Sachverhalts beziehen, bestehen auch zwischen derartigen Bestimmungen denotative Beziehungen, die sich textuell als Kohärenzbeziehungen auswirken. Eine vollständige kohärente Darstellung einer Regelung für den konkreten Fall ergibt sich in solchen Fällen allerdings trotzdem nicht: Wer wissen will, was für ihn gilt, wird auf verschiedene Erlasse verwiesen.

\subsection{Regelungen und Regelungsbereiche}

Dass Regelungen zu einem bestimmten Sachbereich auf verschiedene Erlasse verteilt werden, ist einmal gesetzgeberisch begründet: Im modernen Rechtstaat stützt sich ein Erlass immer auf eine bestimmte eingeschränkte Kompetenz. Diese Kompetenzfestlegung impliziert, dass in einem Erlass ein bestimmter Sachbereich oder Handlungskomplex nur unter einem bestimmten eingeschränkten Aspekt, nicht umfassend geregelt wird. Gewisse Bereiche, die im Alltag als zueinander gehörig empfunden werden, werden deshalb oft in unterschiedlichen Erlassen geregelt. Als Beispiel könnte man die Lebensmitteldeklaration in der Schweiz nennen. Diese stützt sich einerseits auf die Lebensmittelgesetzgebung, andererseits, wenn es um Bio-Produkte geht, auf spezielle Bestimmungen aus dem Landwirtschaftsrecht. Wer die Deklaration für einen Bio-Joghurt verfasst, muss sich also gleichzeitig nach verschiedenen Gesetzgebungsbereichen richten. Ein weiteres Beispiel ist die Arbeitssicherheit. Diese ist im Schweizer Bundesrecht einmal im Zusammenhang von "Unfallverhütung" durch Vorschriften für die Gestaltung von Arbeitsplätzen, ferner im Zusammenhang der "Unfallbekämpfung" als besondere Anforderungen an technische Einrichtungen und Geräte und gesondert davon durch Strahlenschutzvorschriften und schließlich durch Beschäftigungs- und Arbeitszeitvorschriften im Arbeitsgesetz geregelt. Der Arbeitgeber/die Arbeitgeberin muss also, wenn er Personen in einem bestimmten einzelnen Bereich beschäftigt, für einen Handlungskomplex, der für ihn möglicherweise eine Einheit bildet, gleichzeitig eine Vielzahl unterschiedlicher Erlasse beachten. 


\subsection{Kompetenzdelegationen und Stufen der Gesetzgebung}

Die Kompetenz, Regelungen zu erlassen, ist im modernen europäischen Staat ferner gewöhnlich auf verschiedene Behördenstufen verteilt. Das Parlament als Legislative erlässt die grundlegenden Bestimmungen in Gesetzen. Die Regierung als Exekutive erlässt zu diesen Gesetzen die für den Vollzug notwendigen Ausführungsbestimmungen oder zusätzliche Bestimmungen, soweit das Parlament ihr die Kompetenz dazu erteilt. Die Regierung kann in gewissem Rahmen die Konkretisierung einzelner Bestimmungen untergeordneten Institutionen (Ministerien, in der Schweiz Departementen oder "Bundesämtern“) übertragen, vor allem wenn es sich um sich häufig ändernde technische Einzelheiten handelt. Die Institutionen, die diese Bestimmungen auszuführen haben, sorgen schließlich mittels Weisungen für eine einheitliche Durchführung der Bestimmungen. In dieser Weise sind Grundzüge und Einzelheiten zu bestimmten Regelungen auf verschiedene Texte verteilt. Wer eine Regelung in ihren Einzelheiten erkennen will, muss deshalb mehrere unterschiedliche, aber aufeinander bezogene Texte lesen.

Ein einfaches Beispiel liefern die beiden folgenden Bestimmungen aus der Gewässerschutzgesetzgebung. Artikel 14 Absatz 4 des Gewässerschutzgesetzes begrenzt die Nutztierhaltung auf Landwirtschaftsbetrieben aus gewässerschutztechnischen Gründen. Die Artikel 22-24 (und weitere nachfolgende Artikel) der dazugehörigen Gewässerschutzverordnung präzisieren verschiedene im Gesetz genannte Bedingungen wie "Betrieb mit Nutztierhaltung", "Düngergrossvieheinheit" oder den Umfang des im Gesetz genannten "ortsüblichen Bewirtschaftungsbereichs":

\footnotetext{
Art. $14 \quad$ Betriebe mit Nutztierhaltung

${ }^{1}$ Auf jedem Betrieb mit Nutztierhaltung ist eine ausgeglichene Düngerbilanz anzustreben.

${ }^{2}$ Hofdünger muss umweltverträglich und entsprechend dem Stand der Technik landwirtschaftlich oder gartenbaulich verwertet werden.

${ }^{3}$ Im Betrieb müssen dafür Lagereinrichtungen mit einer Kapazität von mindestens drei Monaten vorhanden sein. Die kantonale Behörde kann jedoch für Betriebe im Berggebiet oder in ungünstigen klimatischen oder besonderen pflanzenbaulichen Verhältnissen eine grössere Lagerkapazität anordnen. Für Ställe, die nur für kurze Zeit mit Tieren belegt sind, kann sie eine kleinere Lagerkapazität bewilligen.
} 


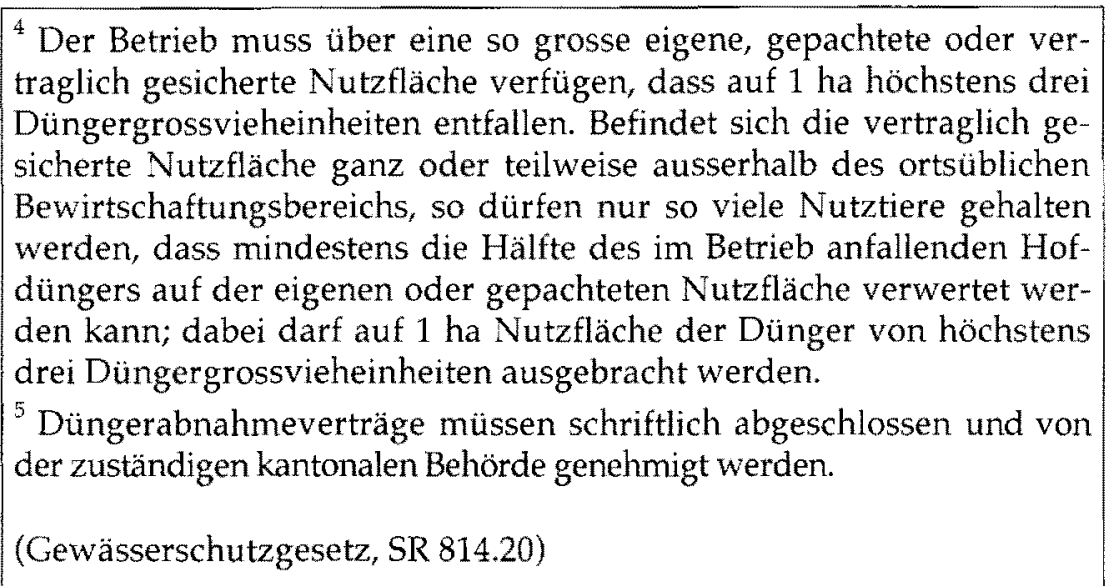

\section{Art. 22 Betriebe mit Nutztierhaltung}

Als Betriebe mit Nutztierhaltung (Art. 14 GSchG) gelten:

a. landwirtschaftliche Betriebe und Betriebsgemeinschaften mit Nutztierhaltung;

b. übrige Betriebe mit gewerblicher Nutztierhaltung; ausgenommen sind Betriebe mit Zoo- und Zirkustieren sowie mit einzelnen Zug-, Reit- oder Liebhabertieren.

Art. 23 Düngergrossvieheinheit (DGVE)

Für die Umrechnung der Nutztiere eines Betriebs auf DGVE (Art. 14 Abs. 4 GSchG) ist ihre jährlich ausgeschiedene Nährelementmenge massgebend. Diese beträgt für eine DGVE $105 \mathrm{~kg}$ Stickstoff und $15 \mathrm{~kg}$ Phosphor.

\section{Art. 24 Ortsüblicher Bewirtschaftungsbereich}

${ }^{1}$ Die vertraglich gesicherte Nutzfläche befindet sich ausserhalb des Bewirtschaftungsbereichs (Art. 14 Abs. 4 GSchG), wenn sie vom Stallgebäude, in dem der Hofdünger anfällt, weiter als $6 \mathrm{~km}$ Fahrdistanz. entfernt ist.

${ }^{2}$ Die kantonale Behörde kann diese Begrenzung unter Berücksichtigung der ortsüblichen Bewirtschaftungsverhältnisse herabsetzen oder um höchstens $2 \mathrm{~km}$ erhöhen.

(Gewässerschutzverordnung, SR 814.201) 
Nur wenn man die Gesetzesbestimmung und die ergänzenden Verordnungsbestimmungen zusammen berücksichtigt, kennt man die ganze Regelung über die Anforderungen an den Gewässerschutz bei Betrieben mit Nutztierhaltung.

Vollzugsverordnungen $\mathrm{zu}$ einem Gesetz konkretisieren entsprechend oft der Reihe nach einzelne Details der verschiedenen Gesetzesbestimmungen. Sie weisen entsprechend auch keine in sich kohärente Struktur auf, die Verordnungsbestimmungen sind nur als Zusatzregelung zu den einzelnen Bestimmungen des Gesetzes verstehbar. Von der Textlogik her betrachtet müssten eigentlich die Gesetzesbestimmungen und die dazugehörigen Verordnungsbestimmungen spaltenweise nebeneinander dargestellt werden, damit die Zusammenhänge auch textlich erkennbar sind.

\section{Fazit: Funktion, Form und Verstehen}

Erlasse sind, so zeigen die voranstehenden Überlegungen, Texte, welche die Erwartungen und Anforderungen an optimal kohärente, kooperative und verständliche Texte nur teilweise oder gar nicht erfüllen. Es wurde versucht zu zeigen, dass diese Unterschiede in den besonderen institutionellen und funktionalen Bedingungen und Anforderungen für Erlasstexte begründet sind:

Erlasse sind nicht Texte, die zur fortlaufenden Lektüre bestimmt sind und aus denen man sich durch diese fortlaufende Lektüre ein mentales Modell eines Wirklichkeitsausschnitts konstruiert. Der Zweck von Erlassen besteht nicht darin, einen Sachbereich darzustellen, sondern darin, dafür möglichst präzis und eindeutig Regelungen festzulegen. Es soll in einem Erlass nicht mehr und nicht weniger gesagt werden, als was als Regelungsinhalt nötig ist. Die Grundsätze der normativen Relevanz und der Identität von Regelung und Bestimmung haben den Sinn, jede einzelne Bestimmung eines Erlasses ganz klar als eine für sich anwendbare selbstständige Regelung identifizierbar zu machen und jedes Element auszuschließen, das diesem Zweck zuwiderlaufen könnte. Jede Gesetzgebung ist auch in ihrem Inhalt und ihrer Reichweite rechtlich eingeschränkt. Das führt dazu, dass die verschiedenen normativen und kommentierenden Aussagen zu einem bestimmten Problem vielfach auf verschiedene Texte verteilt sind.

Gesetzestexte erfordern in mancher Hinsicht eine andere Art des Rezipierens und Verstehens als fortlaufende Alltagstexte. Lasser formuliert als Grundstrategie des Textverstehens: 
,... dass neue Information laufend mit der bereits verarbeiteten integriert wird. Dieser Prozess generiert ein zunehmend umfangreicheres mentales Textmodell mit vielfältigen Beziehungen zwischen den im Text vorkommenden Referenten. ... Bei erfolgreichem Verstehen entsteht beim Leser ein kohärentes mentales Textmodell." 14

Eine derartige Erwartung an das Textverstehen kann von Gesetzestexten fast unweigerlich nur enttäuscht werden. Auch andere Anforderungen an das Verstehen können bei Gesetzestexten nur bedingt erfüllt werden, etwa jene, dass man bei erfolgreichem Verstehen das Wesentliche erkennen und umfangreichere Passagen zusammenfassen können soll (als vereinfachte Wiedergabe des rekonstruierten Text-Weltmodells). Wenn oben gesagt wurde, dass alle Bestimmungen eines Gesetzes sozusagen gleich wichtig sind, dann heißt das auch, dass gerade solche Leistungen nicht möglich sind. Und nicht zuletzt gilt: "Recht will formal sein"15. Das einzige, was die Rechtsanwenderinnen und Rechtsanwender und die Rechtsunterworfenen an Gemeinsamem haben, um sich über das Recht zu einigen, ist der Wortlaut von Erlassbestimmungen als formales Objekt.16 Das heißt: Bestimmungen sind, wenn sie einmal festgesetzt und beschlossen sind, nicht paraphrasierbar, zusammenfassbar, konkretisierbar. ${ }^{17}$

Das Verstehensmodell für gewöhnliche Sachtexte, wie es eben erwähnt wurde, geht von einem dynamischen, in der Zeit ablaufenden Prozess aus. ${ }^{18}$ In diesem Sinn basiert ein Teil der Textformulierungsstrategie auf zeitlichen Erkenntnisprozessen aus Aufbauen von Voraussetzungen als Grundlage des Verstehens, Wecken und Steuern von Erwartungen, Eingrenzen von Fragen und Beantworten von derart eingegrenzten Fragen und Erwartungen. Auch Sachtexte können in diesem Sinne ein gewisses Spannungspotenzial aufweisen. Die relevante Lektüre von Erlasstexten ist nicht in diesem Sinne zeitlich gesteuert, diese können grundsätzlich nicht als spannende Texte aufgebaut werden (auch dann, wenn sie auf Grund der Linearität von Sprache im zeitlichen Ablauf gelesen werden). Die relevante Lektüre besteht darin, dass man im Text für konkrete Sachverhalte - Handlungen, Objekte usw. -

14 LASSER, Verständliche Gesetze - eine Utopie? (Fn. 1), 51.

15 STANLEY FISH, Recht will formal sein, in: KENT D. LERCH (Hrsg.), Recht verstehen. Ver ständlichkeit, Missverständlichkeit und Unverständlichkeit von Recht. Berlin 2004, 85-137.

${ }^{16}$ Wie das Problem der Auslegung und die Vielfalt von Auslegungen in strittigen Fällen zeigt, ist gerade der Inhalt nicht dasjenige, worüber man sich einig ist.

17 Darin besteht die Verwandtschaft zwischen Gesetzestexten und Lyrik.

18 Heinemann. \& Heinemann weisen freilich darauf hin, dass es für ein und denselben Text unterschiedliche Lesestrategien gibt; vgl. HEINEMANN/HEINEMANN, Grundlagen der Textlinguistik (Fn. 9), 171 ff. Das oben Gesagte gilt also vor allem für ein sozusagen prototypisches Lesemuster, wie es aber in der Forschung eigentlich als normal vorausgesetzt wird. 
überprüft, ob und inwiefern dazu im Erlass eine Vorschrift besteht. Der ganze Erlass muss sozusagen für eine derartige Überprüfung gleichzeitig dem Bewusstsein präsent sein.

Um einen Vergleich zu geben (den man nicht strapazieren darf): Ein fortlaufender gewöhnlicher Prosatext ist mit einem Musikstück zu vergleichen, das im zeitlichen Ablauf Themen entwickelt und abwandelt; seine Organisation ist eine Organisation der Zeit. Das Rezeptionserlebnis besteht in der Wahrnehmung dieses zeitlichen Ablaufs. Ein Erlass macht stattdessen eine Auslegeordnung: Alle Gegenstände werden auf einem Tisch möglichst übersichtlich ausgebreitet, damit sie je nach Bedarf verwendet werden können. Dies stellt eine statische Organisation dar, in der die einzelnen Gegenstände zeitlos gleichzeitig präsent und gültig sind.

Die Vorstellung einer "Auslegeordnung“ bedeutet immerhin, dass auch Erlasstexte in ihrer Textstruktur optimierbar sind, selbst wenn sie nicht in allem den Rezeptionsregeln von Sachtexten gehorchen. Wichtig in einer Auslegeordnung ist, dass man das gesuchte Objekt möglichst einfach finden kann. Dazu muss ein Text eine innere logische Ordnung haben. Diese Ordnung wird mit Vorteil den üblichen Regeln der Textorganisation folgen; dazu kommen noch, wie in allen Textsorten, konventionelle Ordnungsprinzipien. Nicht nur muss diese Ordnung nach einer Logik der Gruppierung der einzelnen Gegenstände erfolgen. Auch in Erlasstexten werden durch das Vertexten zwischen den einzelnen Elementen inhaltliche Beziehungen gestiftet, aus denen Schlussfolgerungen gezogen werden, die über die explizit formulierten Informationen hinausgehen und die auch durch das lineare Nebeneinander im Text beeinflusst werden. Eine Aufgabe der Gesetzesredaktion ist daher auch, derartige innere implikative Beziehungen bei der Gestaltung korrekt und ökonomisch in eine geeignete äußere Sprach- und Textform zu bringen. 